Revista LIDER, 37(22), 2020, pp. 63-76

eISSN: 0719-526

ISSN: 0717-0165

\title{
Cuando el desarrollo es una visión de pasado: extractivismo minero y conflictividad ambiental
}

\section{When development is a vision of the past: mining extractivism and environmental conflict}

\section{Daniela Escalona Thomas}

Universidad Academia de Humanismo Cristiano,Chile.daniela.escalonathomas@gmail.com

\section{RESUMEN}

El desarrollo siempre es una visión, imagen o expectativa del futuro. Sin embargo, en territorios producidos por el extractivismo minero, donde las condiciones ambientales que permiten o permitieron ciertos modos de vida son destruidas, aquella situación es significada por las comunidades locales como una ruptura de las proyecciones y posibilidades de desarrollos futuros. A través del estudio de los casos de la Comunidad Aymara de Cancosa y en la localidad de Quillagua, sobre diferentes nociones y perspectivas conceptuales sobre desarrollo y territorios se identifica en sus narrativas como las condiciones de vida pasada se resignificaron para dar sentido y contenido a la definición actual del desarrollo. Se establece que el extractivismo minero produjo un daño ambiental que limita radicalmente las posibilidades de desarrollo actuales y futuras, produciendo que sus habitantes perciban que las condiciones ambientales y sociales que tuvieron en el pasado constituyan el único imaginario de desarrollo posible.

Palabras clave: desarrollo; extractivismo; progreso; conflictos ambientales

ABSTRACT

Development is always a vision, image or expectation of the future. However, in territories produced by mining extractivism, where the environmental conditions that allow or permitted certain ways of life are destroyed, that situation is signified by local communities as a breakdown of projections and possibilities for future developments. Through the study of different notions and conceptual perspectives on development and territory, past life conditions are identified that were resignified to give meaning and content to the definition of development in the cases of the Aymara Community of Cancosa and in the town of Quillagua. We found that mining extractivism produced environmental damage that radically limits current and future development possibilities, causing its inhabitants to perceive that the environmental and social conditions they had in the past constitute the only imaginary of possible development.

Key words: development; extractivism; progress; environmental conflicts

Recibido: 8 de octubre de 2020. Aceptado: 9 de noviembre de 2020.

DOI: $10.32735 / S 0719-5265202037176$ 


\section{Introducción}

Desde mediados del siglo XX la irrupción del desarrollo modernizador, y las nociones relacionadas con él -como progreso, proyecto, evolución o avance-, así como todos los planteamientos críticos que se han construido sobre este concepto, hacen referencia a la idea de porvenir y de futuro (Chambers, 1997; Múnera, 2007). Esto también se replica en las visiones, imaginarios o acciones conducentes al desarrollo, asociándolas siempre a algo que debiera ocurrir.

La expansión del extractivismo en América Latina a partir de la década de los noventa, responde a un proceso que ocurre en las regiones con disponibilidad de recursos naturales y necesidad de inversiones extranjeras, con el propósito de incrementar el crecimiento económico como elemento fundamental para alcanzar el desarrollo (Escalona, 2020), sin embargo ha demostrado un impacto ambiental, social y cultural en los territorios donde se instala que, a pesar del crecimiento económico que ha producido a escala nacional y/o regional (Daher, 2003; Garcés, O'Brien \& Cooper, 2010), es fuertemente cuestionado por las comunidades locales que sufren las externalidades de este modelo (Alimonda, 2011). Es por ello que el daño ambiental grave y en ocasiones irreparable y el quiebre de los tejidos sociales producidos por el extractivismo minero en pequeñas localidades (Alimonda, $2011 \mathrm{y}$ 2015, Bebington, 2007), implican una ruptura profunda en el devenir territorial de una comunidad y su espacio (Escalona, 2014; 2020) y en los sujetos que viven en aquellos lugares.

Esta situación en Chile, se refleja en que el crecimiento y reconfiguración de las exportaciones (Camus \& Hajek, 1998; Rehner, Baeza \& Barton, 2015) así como la expansión territorial de las intervenciones (Garcés, 2003; Garcés, Cooper \& Barros, 2007) ha dejado impactos en gran parte del territorio nacional (Padilla \& San Martín, 1995). Se reconoce por tanto, que el extractivismo minero es un modelo hegemónico con una doble escala de acción: a escala nacional puede efectivamente generar crecimiento económico, sin embargo a una escala local -que es donde se producen los conflictos ambientales-, la situación sobre el desarrollo e incluso sobre el crecimiento económico es ambigua, puesto que los beneficios del extractivismo no se muestran para todos de la misma manera (Escalona, 2020).

La minería extrae recursos y contamina grandes extensiones de territorio desmejorando las condiciones ambientales, en la mayoría de los casos de forma irreparable. Dentro de las múltiples consecuencias del daño ambiental, se identifica en este artículo, como la transformación del territorio modifica los imaginarios y proyecciones de desarrollo de sus habitantes. En los casos que se presentan en este artículo, la Comunidad Aymara de Cancosa y la localidad de Quillagua, esta situación se hizo evidente, puesto que sus habitantes reconocen y significan el desarrollo únicamente en el pasado, en anteriores formas de vida y de producción, que les 
permitían vivir en aquellos territorios que hoy muestran condiciones ambientales que no admiten aquella rememorada forma de vida pero tampoco alguna otra imaginable. Las condiciones ambientales actuales, destruyeron las posibilidades de desarrollo futuro, puesto que no solo transformaron materialmente el territorio sino también las relaciones que lo constituyen, incluidos sus representaciones y significados.

Los resultados que se presentan en este artículo, forman parte de una investigación sobre narrativas de desarrollo en contexto de conflicto ambiental con la minería. La noción de desarrollo como visión de pasado, surgió de las narrativas de la mayor parte de los dirigentes entrevistados. Específicamente fueron los dirigentes históricos y de edad adulta los que asociaron el desarrollo al pasado, puesto que ellos experimentaron aquella forma de vida previa a los impactos ambientales de la minería.

La información se sistematizó a partir de la documentación de los casos, entrevistas a dirigentes históricos y actuales, asi como observación de campo. Considerando que los casos analizados constituyen comunidades con pocos habitantes, fue posible entrevisar a la mayor parte de los dirigentes. El enfoque metodológico incluyó análisis de estructuras narrativas y de argumentación con las cuales analizar el campo discursivo, combinando los enfoques de argumentación política (Fairclough y Fairclough, 2012).

\section{Desarrollo en el tiempo no es desarrollo en el espacio}

Las distintas acepciones bajo las cuales se ha analizado y discutido el concepto desarrollo, desde las más simples; desarrollo como visión, como proceso histórico o como acción (Thomas, 2000), hasta llegar a establecerlo como discurso hegemónico e instrumento político e ideológico para la transformación territorial, para el control y la gobernanza (Cowen \& Shenton, 1996; Peet \& Watts, 2004), lo establecen como una condición mejor que la actual, es decir, el desarrollo es una situación espacio-temporal que va a ocurrir. Sin embargo, estas dimensiones -espacio y tiempo- se han distanciado, ya que territorios avanzados y atrasados conviven. Esta situación fue reconocida como estructural de este modelo de desarrollo económico capitalista, por parte de las teorías críticas surgidas en los años setenta en Latinoámerica, pero que aplicaba para otros territorios subalternos o dominados. Dicha tensión espacio temporal es, a la vez, una limitante en la propia comprensión del concepto, puesto que, si bien el desarrollo se sitúa por definición en el futuro o porvenir, los procesos territoriales para alcanzar este estado o momento, están mediados por estrategias que impactan otros territorios y por tanto, otras trayectorias desarrollistas. De este modo lo que se conjuga es más bien una diversidad de procesos hacia múltiples formas de desarrollo. 
El desarrollo como progreso (Cowen y Shenton, 1996) es definido como el proceso "natural" o inmanente (Thomas, 2000) que comprende a la sociedad humana como concebida para transitar inexorablemente hacia un estado cada vez mayor de civilización. Este desarrollo constante ha ocurrido en la humanidad desde siempre, mucho antes de que el desarrollo fuera "un problema", esta idea se ajusta a una de las definiciones más simples y mayormentecitadas que es la de Chambers (1997) quien menciona que el desarrollo es un cambio para mejor. Esta noción se asocia al concepto de progreso, puesto que hay siglos de avance que anteceden al desarrollo concebido desde la industrialización capitalista. De acuerdo a Cowen y Shenton (1996) solo el capitalismo y su producción de pobreza, desempleo y miseria obligan a una actividad constructiva e intencional del desarrollo.

Thomas (2000) enuncia algunas consideraciones para aproximarse a una definición más compleja, en primer lugar, que el desarrollo es multidimensional, ya que implica un cambio sobre varias dimensiones, no solo una; segundo que es progresivo , que el desarrollo es un proceso de cambio que se produce sobre las modificaciones ya realizadas, es decir se supera a sí mismo en una continua transformación, y tercero, que el desarrollo es un proceso de cambio que ocurre al mismo tiempo tanto en el nivel social o colectivo como individual, todo esto ocurre más alla de cualquier ideología o modelo.

Lo dicho anteriormente contrasta con el desarrollo tal y como es concebido en la economía capitalista en su vertiente neoliberal, puesto que los Estados han debido tomar este discurso y adaptarlo a sus realidades políticas, pero no geográficas. Esta visión desarrollista constituye una «refracción» de la ideología y cultura del progreso, puesto que es un proceso que concretiza ciencia, tecnología y producción de riqueza -expresiones de la capacidad humana de comprensión del mundo físico y social-, para manipularlo y organizarlo para su beneficio (Preston, 1999), esta perspectiva derivada de la economía neoclásica, se vincula discursivamente a la modernización.

En la enorme diversidad de planteamientos críticos que surgen frente al desarrollo hegemónico, una gran parte se basan en la naturaleza reduccionista y economicista del concepto, en ellas aparece como central el hecho de que la propia noción de desarrollo se llega a establecer como sinónimo de crecimiento económico y que "esta similitud entre las nociones ha tenido graves implicaciones para los procesos de la sociedad, ya que mientras la primera se refiere a expansión la segunda solo a la adición de materialidad" (Svampa y Antonelli, 2009, p. 72), los planteamientos críticos más extendidos se refieren a que el nivel de desarrollo es una condición impuesta y relacionada con el lugar que ocupa cada economía en el modo global de producción capitalista. 
Esta situación ha llevado a que actualmente este discurso sea fuertemente cuestionado por las comunidades locales, evidenciando que, de acuerdo a ciertas perspectivas, el desarrollo económico es ante todo una lucha contra la pobreza y por satisfacer las necesidades de la población, y que por tanto debiese estar subordinado a la voluntad de las poblaciones locales (Fontaine, 2003).

Puesto que el nivel de desarrollo es una condición impuesta de acuerdo a la posición en la economía capitalista, el crecimiento económico experimentado por algunos territorios implica el empobrecimiento de otros. La producción de esta desigualdad espacial, derivada del modelo extractivo, es lo que define los territorios que se presentan en este documento, puesto que se constituyen, como territorios con trayectoria propia que es interrumpida por proyectos de explotación minera que no los benefician puesto que las riquezas se derivan a otros sitios, no asumen política $o$ económicamente sus externalidades negativas, las que son transferidas a estos terriorios marginales. Esta situación, modifica el medio ambiente y las trayectorias espaciales, pero también los imaginarios y las significaciones que estos habitantes tienen sobre sus territorios.

\section{Deterioro ambiental como limitante de las posibilidades de desarrollo en territorios locales}

El modelo extractivo exportador, constituye el contexto en el cual se producen y construyen los territoritos mineros, que como cualquier territorio condiciona y posibilita el desarrollo. Estos espacios, por una parte materializan el discurso homogeneizante del neoliberalismo $y$ por otra son producto de la instrumentalización del discurso del desarrollo, es decir, los territorios mineros se definen por las relaciones de poder que se expresan tanto simbólica como materialmente en un momento histórico específico (Alimonda, 2011; Romero \& Sasso, 2014).

En Chile, la minería se ampara en una norma que la protege (Código de Minería, 1983) y que cuenta con privilegios territoriales (Código de Aguas, 1981) que ninguna otra actividad económica tiene, es decir, la producción de los territorios mineros en el país es resultado, en primer término, de la acción del Estado que ha institucionalizado la actividad minera, privilegiándola como una decisión económica estratégica (Luna, Padilla \& Alcayaga, 2004). Sin embargo, para las comunidades locales la minería no ha significado el desarrollo que había prometido (Cuenca, 2008), y ello ocurre porque dichas comunidades no sólo son marginadas de los procesos productivos que impulsa la mineria, sino fundamentalmente porque las externalidades negativas que produce los llevan al desplazamiento o la desaparición. 
A escala local es donde se experimentan las consecuencias mas negativas. Desde la dimensión ecónomica, se produce un crecimiento desigual, ya que existe a esta escala una concentración de beneficios en los actores vinculados a la produccion minera, mientras que el resto de la población asume las externalidades negativas. Entre estas externalidades asumidas por comunidades tradicionales especialmente indígenas en el norte del país, se destacan el grave deterioro ambiental, migración, abandono de territorios comunitarios y desaparición de actividades económicas y culturales tradicionales, asi como el daño en el tejidos sociales de estas comunidades pequeñas y cohesionadas (Romero, Mendez \& Smith, 2012; Yañez \& Molina, 2008, 2011; Escalona, 2020). Los conflictos ambientales resultantes (desatados o latentes) significan una ruptura en el devenir de estas comunidades, en sus trayectorias propias hacia desarrollos otros.

Los casos de estudio que hicieron surgir la visión de desarrollo como pasado o en el pasado, corresponden a dos casos emblemáticos de destrucción ambiental ocasionada por la minería en Chile, ambas comunidades indígenas, actualmente experimentan los efectos más perversos ocasionados por la gran minería; pérdida de recursos hídricos, contaminación de los mismos, asi como una degradación general de sus ecosistemas que no les permiten hoy las ancestrales y tradicionales prácticas productivas (Romero, Mendez \& Smith, 2012; Yañez \& Molina, 2008).

Las poblaciones que habitan estos lugares, se situaron allí gracias a una relación estrecha con aquellas condiciones naturales, como campesinos y/o indígenas, dependen de los ambientes para subsistir. Por lo tanto, el daño de estos ecosistemas constituye una ruptura en el devenir territorial que puede llegar a ser tan determinante para estas comunidades tradicionales, ya que la naturaleza constituyen no solo su espacio de habitar, sino también es fuente de su medios de producción y por tanto, de sus medios de vida, es por esto que los significados que la población le da al deterioro ambiental pueden llegar a ser tan profundos, "Bueno, yo lo sentí como el peor puñal que nos pudieron haber dado" (Dirigenta Quillagua, 12 de julio de 2015).

La capacidad destructiva del extractivismo se ejerce con una potencia que bloquea la posibilidad misma del desarrollo local, sumiendo a los territorios de la periferia en la desorganización de sus sistemas productivos y sociales, produciendo y reproduciendo con ello la heterogeneidad estructural de América Latina (Alimonda, 2015). De este modo, el extractivismo como "desarrollo de fuerzas productivas" se instala a modo de enclaves, que se hacen ingobernables por los Estados nacionales (Alimonda, 2015) así como imposibles de asimilar por las sociedades locales (Bebbington, 2007), por la desproporción de escalas que bloquea la transferencia de cualquier estimulo hacia un desarrollo consecuente con los territorios. Así, el resultado no es el progreso de los territorios, sino la destrucción 
ambiental, política y social de las comunidades locales, reproduciendo la desigualdad espacial y haciendo territorios económicamente dependientes (Alimonda, 2015).

\section{La muerte del fértil oasis de Quillagua}

El primer caso corresponde a la localidad de Quillagua, localizada en el curso medio del rio Loa. Compuesta por unas 102 personas según el censo de 2002, integran una comunidad agrícola tradicional, constituida por 76 propiedades agrícolas, que a comienzos de siglo inicia un proceso de re-etnificación aymara (Mc Phee, 2010). Sin duda, la tensión territorial que se ha producido en la cuenca del Loa se relaciona con la minería de CODELCO, esta tensión se ha identificado en base a dos problemáticas fundamentales; la necesidad de la empresa minera de controlar las aguas superficiales y subterráneas para asegurar el proceso productivo, esto con la consecuente reducción del volumen de agua para las otras actividades, principalmente el riego, expulsando a la población interior a las ciudades; en segundo lugar la contaminación de las aguas producto de la infiltración del tranque de relaves Talabre de Codelco (Larraín y Poo, 2010), situación que se refleja en lo vivido por esta localidad.

A esto se sumó el Código de Aguas de 1981, que significó para las comunidades atacameñas una reducción ostensible de los derechos de aprovechamiento al permitírseles a los agricultores inscribir sólo una parte de las aguas históricamente utilizadas (Molina, 2006). Para el caso de Quillagua, los canales de riego conducían un caudal de 350 litros por segundo para riego el de las 400 hectáreas de cultivos. Terminada la inscripción de los derechos de aguas, la comunidad solo conservó 120 litros/segundos, es decir disminuyó el 66\% del total de los derechos de aprovechamiento de que disponían antes de la inscripción.

El colapso de Quillagua comenzó a manifestarse en el año 1997, cuando a causa de las inundaciones se produce el primer evento de contaminación de las aguas del río Loa (Barros \& Rowlands, 2008), sin embargo, la autoridad ambiental no ejerció ninguna acción para revertir la situación que terminó por hacer desaparecer la agricultura producto dexantato, químico utilizado en la minería de cobre (Mc Phee, 2010)

Se empezaron a morir los animales, entonces ahí, empezaron a ver el agua, claro el agua estaba contaminada, imagínate se contaminaron las caletas en toda la costa de Iquique (Dirigenta Quillagua, entrevista septiembre 2017).

En ese momento los pocos derechos de agua de la comunidad no tenían valor, puesto que no podian ser ejercidos, así que la mayor parte de los comuneros los vendió a un menor precio a SOQUIMICH, empresa también estatal. 
A causa de la contaminación de las aguas, el abandono de las tierras, la merma en los recursos forestales, la aceleración del proceso de desertificación y la pauperización rural generalizada (Larraín y Poo, 2010), se ha producido un fuerte proceso de migración, envejecimiento de la población y con ello una desintegración la vida social y comunitaria. A pesar de ello se produjo, paralelamente al conflicto, un proceso de re-etnificación (Mc Phee, 2010), que les ha permitido recuperar parte de los derechos de agua.

A pesar de que la destrucción ambiental constituye una ruptura insoslayable en el devenir territorial, modificando sus imaginarios y visiones futuras, esta comunidad actualmente, continua resistiendo en este territorio, buscando nuevas posibilidades para permanecer asi como lo han hecho las comunidades aymaras que los precedieron.

\section{El despoblamiento de la Comunidad Aymara de Cancosa}

El caso de la comunidad aymara de Cancosa frente a BHP Billiton y su proyecto Cerro Colorado, tuvo un proceso distinto pero con un resultado similar. La comunidad estableció una relación con la empresa minera, en 1991 al momento que arrendaron el fundo Huatinja (DL No67, 2001), de propiedad colectiva, para la construcción de pozos que permitieran a la empresas extraer los recursos hídricos para la faena minera por 30 años, que dista 50 kilimetros del lugar (Mamani, 2008). El problema se generó cuando la comunidad se dió cuenta que producto de la extracción subterránea los bofedales altoandinos situados en Pampa Lagunillas, comenzaron a desecarse. Esto los llevó a iniciar denuncias públicas y un proceso jurídico, que se cierra debido a una negociación privada que sostienen con la empresa. Adicionalmente y motivado tanto por la fiscalización ambiental como por el acuerdo con la comunidad, la empresa se compromete a reparar el daño ambiental, lo que hasta hoy no se ha cumplido satisfactoriamente.

De acuerdo a sus propios registros, se compone de más de 80 familias, unas 350 personas, sin embargo, de acuerdo al Censo de 2002, solo 26 de ellas habitan el territorio. Aunque en la visita a terreno se constató que los habitantes permanentes no superan las 6 personas a lo que se suma unas 20 personas que visitan el lugar para cuidar su ganado y sus plantaciones de quínoa (Romero-Toledo et al., 2017). Los comuneros atribuyen este abandono a diversos factores históricos (Gundermann y Vergara, 2009), pero consideran que el impacto de la minería y el dinero recibido en la negociación constituyó el último impulso para su migración a ciudades cercanas, como Alto Hospicio, Iquique, Pica y Mamiña, donde se concentran actualmente. 
Actualmente los miembros de la comunidad indígena se encuentran disgregados, pero aún siguen teniendo un fuerte aprecio de la vida comunitaria y los valores colectivos como solidaridad, confianza, participación. Señalan que, si bien la forma de vida tradicional aymara ya no es posible, continuarán trabajando por mantener sus vínculos sociales, así como su relación con la sociedad chilena que les han permitido acceder a educación y a bienes materiales, sin embargo, consideran que el acceso material que les posibilitó la negociación con la empresa, no ha significado para ellos un desarrollo de la comunidad (Escalona, 2020).

\section{Sin proyecto de futuro no es posible significar el desarrollo}

Los territorios donde las faenas mineras se instalan, donde extraen agua o bien donde depositan sus desechos, son sitios que se caracterizan por un evidente daño ambiental. Cuando las condiciones ambientales son destruidas de forma temporal o irreversible las posibilidades de desarrollo se restringen. Las actuales condiciones ambientales de los territorios mineros descritos, así como de muchos otros, dificultan la posibilidad de pensar o repensar un futuro, puesto que no cuentan con las condiciones mínimas para un nuevo proyecto territorial.

Las apreciaciones de la totalidad de los entrevistados sobre el daño ambiental, evidencian la transformación radical que significó el impacto de la mineria, "yo creo que eso no se puede arreglar" o "vino la contaminación del río Loa y ahí murió todo", donde, sin duda, el elemento central dentro de los impactos ambientales lo constituye la pérdida de los recursos hídricos, tanto en calidad como en cantidad, lo que es considerado una limitante para imaginar nuevas posibilidades en el futuro, especialmente en zonas áridas que es donde se ubican estas localidades con una historia vinculada a la producción agrícola.

Ante esta experiencia de ruptura territorial, se manifiesta en los habitantes una dificultad para imaginar o proyectar el desarrollo en estos territorios con las actuales condiciones ambientales, por lo cual, las representaciones del desarrollo, sólo son encontradas en su propia experiencia. De este modo, la forma que adquieren estas descripciones de la vida pasada, son reconocidas por los sujetos como situaciones prósperas y desarrolladas.

En su pasado, reconocen y enuncian una escala de trabajo y un equilibro entre las condiciones ambientales, económicas, sociales y culturales, elementos necesarios para una vida satisfactoria: "Nosotros aquí teníamos de todo" (Dirigente Quillagua, entrevista septiembre de 2015). Visualizando las potencialidades territoriales asociadas fuertemente a las características ambientales de aquella época: "En mis tiempos Quillagua era un pueblo desarrollado" (Dirigenta Quillagua, entrevista septiembre de 2015). 
La asociación que se hace del desarrollo con la forma de vida pasada, se configura a su vez como una crítica a los valores impuestos por el modelo neoliberal, tales como el consumo, la preocupación y el dinero, "la gente estaba tranquila, seguramente no tenía todas las comodidades, cierto, no tenía nada, pero la gente vivía feliz" (Dirigente Cancosa entrevista, 21 de julio de 2015). Puesto que las posibilidades generadas desde el modelo neoliberal, se circunscriben a los beneficios indirectos parciales y desiguales que han recibido (compensaciones económicas por parte de las empresas, bonos y pequeños proyectos estatales).

A pesar de cuestionar los resultados de la llegada de la minería en términos ambientales y económicos, no logran cuestionar con la misma radicalidad, el crecimiento económico como centro de aquel modelo de desarrollo, es decir, a pesar de reconocer las consecuencias del modelo minero extractivo, no cuestionan que lo que está detrás es la búsqueda del crecimiento económico, puesto que a su vez demandan formar parte activa de los proyectos y de sus beneficios.

La falta de medios de producción agrícola en el territorio debido al daño ambiental, ha provocado esta contradicción, puesto que aquella forma de vida, la única que conocen, ya no es posible, por lo que demandan participación en las nuevas dinámicas económicas que les han sido impuestas. No obstante, la minería no se ha configurado como una alternativa en la que ellos sean beneficiarios o al menos sean partícipes. Identifican que en la cadena del desarrollo-progreso ellos se encontrarían en el último eslabón, ya que son los que asumen el costo ambiental, prácticamente sin recibir beneficios.

La gravedad del daño ambiental es percibida no sólo como causante de impactos ambientales con efectos en la economía local, sino que, atenta igualmente contra los vínculos históricos y culturales de una comunidad con su territorio. Esto, no sólo asociado a las condiciones de dependencia y vínculo estrecho entre comunidades tradicionales y su medio ambiente, sino también porque estos ecosistemas y sus componentes representan -especialmente en el caso de comunidades indígenas - historia, cultura y creencias que les dan identidad y arraigo. Es decir, esta ruptura y la construcción del desarrollo como una visión del pasado, ha significado perder parte de su identidad, ya que una fracción del vínculo que tenían con el territorio también se ha perdido, "Quillagua cuando era Quillagua” (Dirigenta Quillagua, entrevista 13 de julio de 2015).

Las narrativas surgidas en este contexto, cargadas de desesperanza, marginación y abandono, hacen suponer que la tensión territorial asociada al modo de producción capitalista -especialmente en su fase neoliberal-, seguirá siendo motor de conflictividad, ya que no estaría completamente legitimado, no al menos por las poblaciones que éste margina. La situación se complejiza en el caso de las 
comunidades desterritorializadas como las presentadas, donde le desesperanza es aún mayor.

Los resultados expuestos, evidencian una diferencia profunda entre las visiones, expectativas y promesas con las que se articula el discurso de desarrollo de la minería, a pesar de que ha producido expectativas e imaginarios en las propias comunidades locales. Con el tiempo esta brecha no se ha acortado, por el contrario, el aumento de esta distancia ha profundizado la tensión y los conflictos ambientales que se han generado en estos territorios. Si bien los imaginarios de las comunidades locales han estado influenciados por estos discursos hegemónicos, se componen también de elementos identitarios, territoriales e históricos propios.

La fuerte contradicción que se establece entre un discurso de desarrollo único y las derivadas transformaciones y efectos en el territorio local, constituye no sólo una situación de injusticia para aquellas comunidades que asumen sus externalidades, sino que, en términos ambientales, es una hipoteca de futuro para la sociedad en su conjunto, puesto que se están deteriorando los ecosistemas que podrían resultar en nuevos y posibles desarrollos futuros.

\section{Reflexiones finales}

Las visiones de desarrollo de las comunidades afectadas por la minería, entregaron reflexiones que vienen a romper con el paradigma del desarrollo como una visión territorial del futuro, como un proyecto posible o una meta que alcanzar. Ya que cuando no existen proyectos que configuren una visión del porvenir debido a las condiciones materiales perdidas, solo en el pasado se puede visualizar el imaginario de un desarrollo que sí fue posible.

La percepción de los sujetos en relación a las visiones de desarrollo futuras, deja al descubierto visiones de carácter pesimista con respecto al territorio actual y por venir. En primer lugar, por las sentidas y detallas caracterizaciones de la vida pasada, como una vida mejor y más feliz, que incluye elementos valóricos del territorio como prácticas espaciales que fortalecían las relaciones comunitarias, siempre enfatizando en el pasado, implícitamente evidenciando la ruptura con la situación actual.

Los habitantes de estas comunidades están de acuerdo con que el crecimiento económico es necesario para alcanzar el desarrollo, puesto que por una parte, manifiestan la productividad como el camino para el desarrollo y por otra parte, su visión de desarrollo pasado está relacionada con los medios de producción con que contaban y que perdieron debido al daño ambiental.

Los conflictos provocados por la mineria, constituyen una transformación dramática del ambiente físico, así como en las economías locales establecidas o 
heredadas, la destrucción ambiental en estos lugares implica la destrucción de los medios de subsistencia y de permanencia, por tanto, esta irrupción implica cambios no solo en sus prácticas territoriales, sino también en las visiones, imaginarios y concepciones sobre el territorio. Estas sociedales locales marginadas, no están preparadas para confrontar estos "proyectos intensivos en capital y extensivos en espacio" (Alimonda, 2015, p. 130).

El imaginario colectivo de que el tiempo pasado fue un tiempo más desarrollado para sus territorios, muestra que las nociones y significados que se han construido en las comunidads locales, especialmente en contexto de conflictividad ambiental, son narrativas complejas y profundas con respeto al territorio y al desarrollo, instaurando bases aun insuficientemente exploradas, sobre posibilidades o alternativas de otros desarrollos.

\section{Bibliografía}

Alimonda, H. (2011) (ed.) La naturaleza colonizada: ecología política y minería en América Latina. Buenos Aires: CLACSO-CICCUS. Recuperado de: https://mundoroto.files.wordpress.com/2016/11/la-naturaleza-colonizada-ecologc3adapolczadtica-y-minerçada-en-al.pdf

Alimonda, H. (2015) Provocaciones sobre el tema“Extractivismo y Desarrollo" Polis, Revista Latinoamericana, Volumen 14, No 41, 2015, p. 43-57

Barros, A. \& Rowlands, J. (2008) El último oasis del Loa: Agua, Territorio y Sociedad en la comunidad Aymara de Quillagua.Informe a Amnistía Internacional. Recuperado de: https://www.academia.edu/12547117/El último oasis del Loa Agua Territorio y Socie dad_en_la_Comunidad_Aymara_de_Quillagua._Informe_a Amnist\% $\mathrm{C}_{3} \% \mathrm{ADa}$ Internacio $\underline{\text { nal }}$

Bebbington, A. (2007) Una ecología política de la minería y la transformación territorial. En Bebbington, A. (ed.) Minería, movimientos sociales y respuestas campesinas. Una ecología política de transformaciones territoriales. Lima: IEP-CEPES. pp. 23-46. Recuperado de: http://biblio.flacsoandes.edu.ec/catalog/resGet.php?resId=21087

Camus, P. y Hajek, H. (1998) Historia ambiental de Chile. Santiago de Chile. Recuperado de:https://www.ecolyma.cl/documentos/hist_amb_chile_v1.pdf

Chambers, R. (1997) Responsible wellbeing: a personal agenda for development. World Development. Vol. 25 (11), pp. 1743-1754. Recuperado de: https://www.sciencedirect.com/science/article/abs/pii/S0305750X97100018

Cowen, M.P. y Shenton, R.W. (1996) Doctrines of development. London: Routledge. 
Cuenca, L. (2008) Cobre: crecimiento desenfrenado y empobrecedor. Revista de Ecología Política. $\mathrm{N}^{\circ}$ 35, pp.123-126.

Daher, A. (2003) Regiones-commodities. Crisis y contagio en Chile. Revista EURE. Vol. 29 (86), pp. 89-108.

DECRETO CON FUERZA DE LEY N ${ }^{\circ} 1.122$. Chile. Código de aguas. Diario oficial 13 de agosto 1981.

Escalona, D. (2014). Energía termoeléctrica en Chile. Una mirada desde el discurso desarrollista". Revista de Geografía Espacios № 8, pp.49-63.

Escalona, D. (2020) La Negociación en los conflictos ambientales y su implicancia en el desarrollo local: caso de comunidad Aymara en Cancosa. Revista Dialogo Andino $\mathrm{N}^{\mathrm{0}} 61$, pp.81-91.

Fairclough, I. \& fairclough, N. (2012). Political discourse analysis: a method for advanced students, England: Routledge.

Fontaine, G. (2003) Enfoques conceptuales y metodológicos para una sociología de los conflictos ambientales. Guerra, sociedad y medio ambiente, pp. 503-533. Recuperado files/bueros/kolumbien/o1993/12.pdf

Garcés, E; o'brien, J y Cooper, M. (2010) Del asentamiento minero al espacio continental: Chuquicamata (Chile) y la contribución de la minería a la configuración del territorio y el desarrollo social y económico de la Región de Antofagasta durante el siglo XX. Revista EURE. Vol. 36 (107), pp. 93-108.

Garcés, E. (2003) Las ciudades del cobre. Del campamento de montaña al hotel minero como variaciones de la company town. Revista EURE. Vol. XXIX (88), pp. 131-148. Garcés, Cooper \& Barros, 2007

Gundermann, H. y J. Vergara. (2009) Comunidad, Organización y Complejidad Social Andinas en el Norte de Chile. Estudios atacameños. $\mathrm{N}^{\mathrm{o}} 38$, pp.107-126. Recuperado de http://dx.doi.org/10.4067/So718-10432009000200008 LARRAIN, S. y POO, P. (eds.) (2010) Conflictos por el Agua en Chile, Entre los Derechos Humanos y las Reglas del Mercado. Chile: Ediciones Chile Sustentable.

LEY 18.248 Código de Minería. Diario Oficial de la República de Chile. Santiago, Chile. 14 de octubre 1983.

Luna, D., Padilla, C. \& Alcayaga, J. (2004) El exilio del cóndor. Chile: Ediciones OLCA.

Mamani, A. (2008) Desecamiento laguna sector lagunilla en el territorio ancestral de la comunidad de Cancosa. En Bello, A. y Alwyn, J. (comp.) Globalización derechos humanos y pueblos indígenas (pp. 392-397). Temuco: Observatorio de derechos de los pueblos indígenas . 
Mcphee, B. (2010) Conflictos ambientales y respuestas sociales: el caso de reetnificación de la comunidad de Quillagua. Revista Mad (Magister en Análisis sistémico aplicado a la sociedad), $\mathrm{N}^{\circ} 22$, pp. 42-55. Recuperado de: $w w w$.redalyc.org/articulo.oa?id=311224767003

Molina, R. (2006) El rio Loa: Usos y conflictos por el agua en el desierto de atacama. Comunidades indígenas, mineras, ciudades y pueblos. Chile: DGA. Recuperado de:http://www.infoandina.org/es/content/el-rio-loa-usos-y-conflictos-porel-agua-en-el-desierto-de-atacama-comunidades-\%C3\%ADndigenas

Múnera, M. (2007) Resignificar el desarrollo. Medellín: Escuela del hábitat CEHAPUniversidad Nacional de Colombia.

Padilla, C. y San Martin, P. (1995) Conflictos Ambientales: una Oportunidad para la Democracia. Santiago de Chile: OLCA.

Peet, R. y Watts, M.(2004) Liberation Ecologies: Environment, Development, Social Movements (2aed.). London: Routledge.

Preston, P. (1999) Una introducción a la teoría del desarrollo. México: Siglo XXI.

Rehner, J., Baeza, S. y Barton, J. (2015) En hombros de gigantes, una geografía económica del comercio Chile-Asia. Santiago de Chile: Pontificia Universidad Católica de Chile.

Romero Toledo, H.; Videla, A. y Gutierrez, F. (2017) Explorando conflictos entre comunidades indígenas y la industria minera en Chile: las transformaciones socioambientales de la región de Tarapacá y el caso de Lagunillas. Estudios atacameños. $\quad \mathrm{N}^{0} 55, \quad$ pp. 231-250. Recuperado de https://scielo.conicyt.cl/pdf/eatacam/n55/aop1917.pdf

Romero, H. y Sasso, J. (2014) Proyectos hídricos y ecología política del desarrollo en Latinoamérica: Hacia un marco analítico. Revista Europea de Estudios Latinoamericanos y del Caribe, $\mathrm{N}^{\mathrm{O}}$ 97, pp. 55-74. Recuperado de:https://www.erlacs.org/articles/abstract/10.18352/erlacs.9796/

Romero, H.; Méndez, M. \& Smith, P. (2012) Mining Development and Environmental Injustice in the Atacama Desert of Northern Chile. Environmental Justice. Vol. 5(2), pp. 70-76.

Svampa, M. y Antonelli M. (eds.)(2009) Minería transnacional, narrativas de desarrollo y resistencias sociales, Buenos Aires: Editorial Biblios.

Thomas, A. (2000) Meanings and Views of Development. En Allen, T. y Thomas, A. (eds.) Poverty and Development in the 21st Century. Oxford: Oxford University Press.

Yañez, N. y Molina, R. (2008) La gran minería y los derechos indígenas en el norte de Chile. Chile: LOM Ediciones.

Yañez, N. y Molina, R. (2011) Las aguas indígenas en Chile. Santiago de Chile: LOM Ediciones. 